\title{
HIV infection and the immigration in Italy. Consequences on inpatient hospitalizations and Day-Hospital admissions at a metropolitan hospital, during the last nine years
}

Roberto Manfredi

From $16^{\text {th }}$ International Symposium on HIV and Emerging Infectious Diseases

Marseille, France. 24-26 March 2010

\section{Background}

Immigration is a recent phenomenon in Italy, mainly caused by the sudden and unexpected arrival of wawes of foreign citizens, refugees, and individuals escaping from war. This phenomenon is of great concern, due to its serious social-economic and health care impact.

\section{Methods}

A prospective survey of all charts of patients (p) hospitalized or followed on day-hospital (DH) basis at our Infectious Disease ward until end-2008, allowed us to assess the frequency of admission of immigrants from extra-Western Europe (eWE), and to analyze multiple variables related to multiple epidemiological and clinical features.

\section{Results}

The rate of $\mathrm{p}$ immigrated from eWE showed a significant increase among our inpatients, and at a lesser extent and later for DH admissions: $7.7 \%$ and $3.1 \%$ during the year $2000,10.1 \%$ and $4.6 \%$ in $2001,13.2 \%$ and $6.2 \%$ in $2002,17.9 \%$ and $7.9 \%$ in $2003,21.3 \%$ and $8.9 \%$ in $2004,17.7 \%$ and $10,8 \%$ in $2005,17.9 \%$ and $11.3 \%$ in the year $2006,17.3 \%$ and $10.9 \%$ in the year 2007 , up to $17,7 \%$ and $11.4 \%$ in the year $2008(p<.0001$ for inpatients; $p<.001$ for DH p). Over $60 \%$ of $\mathrm{p}$ came from Africa, followed by Eastern Europe, Asia, and CentralSouthern America. When comparing the admission features of WE citizens with those of $\mathrm{p}$ coming from abroad, no differences were found as to duration and intensity of assistance, with HIV disease prevailing among regular admissions (33.6\%), and DH access (30.2\%), followed by acute-chronic hepatitis, pulmonary or other-site tuberculosis, central nervous system and respiratory tract infection, and sexually-transmitted diseases. HIV-infected immigrants were frequently $(>60 \%$ of cases) "AIDS presenters", and less than $5 \%$ of them were already on an antiretroviral therapy upon admission. While the frequency of HIV-associated admissions did not show differences in the considered 9-year period, $p$ from eWE had an increasing frequency of tuberculosis, skin-soft tissue infection, infectious exanthems, gastroenteric-parasitic diseases, and malaria $(p<.05$ to $<.0001)$.

\section{Discussion}

A continued monitoring of this phenomenon is strongly warranted, in order to improve a sustainable social-cultural network, to plan health resource allocation for the next future, and to define adequate and well-targeted prevention and public health measures.

Published: 11 May 2010

doi:10.1186/1742-4690-7-S1-P128

Cite this article as: Manfredi: HIV infection and the immigration in Italy. Consequences on inpatient hospitalizations and Day-Hospital admissions at a metropolitan hospital, during the last nine years. Retrovirology 2010 7(Suppl 1):P128.

Correspondence: Roberto.manfredi@unibo.it

Infectious Diseases, University of Bologna, S. Orsola Hospital, Bologna, Italy 